I0. In respect to the general results, the phenomenon of incompatibility appears to present some analogy to that of so-called antigen-antibody reactions in immunity, and to isoagglutination and isoprecipitation phenomena. Here, however, the incompatibility exists between organs produced side by side in the same flower and which are of close somatic relationship.

\title{
I39（1317）
}

\section{A demonstration of cerebellar and cerebral lesions in dogs.}

By I. Strauss and I. Friesner (by invitation).

[From the Department of Physiology of Columbia University.]

Dr. Pike with his characteristic modesty has asked us to present these dogs. They show lesions which resulted in part from design and in part from accident. The experiments were undertaken in an attempt to study the relations between the internal ear and the central nervous system.

From a clinical point of view otologists look upon the results of labyrinthine stimulation or destruction as divided into two heads:

I. Nystagmus.

2. Vertigo.

The brain pathways which intermediate the nystagmus impulses are well known. On the other hand, those which have to do with vertigo are very little known. Recently the theory has been advanced that the cerebellum intermediates the labyrinthine impulses which ultimately pass to the cerebrum, and have to do with our sense of position.

It was in the attempt to test this theory experimentally that these operations were undertaken. Some of the dogs show the result of injuries to the cerebellar peduncles. In another in attempting to reach the peduncles we accidentally injured the vermis and had to stop. Another dog shows the result of a bilateral cerebellar lesion as well as a vermis lesion. We have but commenced our studies and are indebted for our results largely to the kindly patience and help of Dr. Pike. 